\title{
Herschel-ATLAS: Dust temperature and redshift distribution of SPIRE and PACS detected sources using submillimetre colours ${ }^{\star}$
}

\author{
A. Amblard ${ }^{1}$, A. Cooray ${ }^{1}$, P. Serra ${ }^{1}$, P. Temi ${ }^{2}$, E. Barton ${ }^{1}$, M. Negrello ${ }^{3}$, R. Auld ${ }^{4}$, M. Baes ${ }^{5}$, I. K. Baldry ${ }^{6}$, \\ S. Bamford ${ }^{7}$, A. Blain ${ }^{8}$, J. Bock ${ }^{9}$, D. Bonfield ${ }^{10}$, D. Burgarella ${ }^{11}$, S. Buttiglione ${ }^{12}$, E. Cameron ${ }^{13}$, A. Cava ${ }^{14}$, \\ D. Clements ${ }^{15}$, S. Croom ${ }^{16}$, A. Dariush ${ }^{4}$, G. de Zotti ${ }^{12,21}$, S. Driver ${ }^{17}$, J. Dunlop ${ }^{18}$, L. Dunne ${ }^{7}$, S. Dye ${ }^{4}$, S. Eales ${ }^{4}$, \\ D. Frayer ${ }^{19}$, J. Fritz ${ }^{5}$, Jonathan P. Gardner ${ }^{20}$, J. Gonzalez-Nuevo ${ }^{21}$, D. Herranz ${ }^{22}$, D. Hill ${ }^{17}$, A. Hopkins ${ }^{23}$, \\ D. H. Hughes ${ }^{24}$, E. Ibar ${ }^{25}$, R. J. Ivison ${ }^{25}$, M. Jarvis ${ }^{10}$, D. H. Jones ${ }^{23}$, L. Kelvin ${ }^{17}$, G. Lagache ${ }^{26}$, L. Leeuw ${ }^{2}$, J. Liske ${ }^{27}$, \\ M. Lopez-Caniego ${ }^{22}$, J. Loveday ${ }^{28}$, S. Maddox ${ }^{7}$, M. Michałowski ${ }^{18}$, P. Norberg ${ }^{18}$, H. Parkinson ${ }^{18}$, J. A. Peacock ${ }^{18}$, \\ C. Pearson ${ }^{29,30}$, E. Pascale ${ }^{4}$, M. Pohlen ${ }^{4}$, C. Popescu ${ }^{31}$, M. Prescott ${ }^{6}$, A. Robotham ${ }^{17}$, E. Rigby ${ }^{7}$, G. Rodighiero ${ }^{32}$, \\ S. Samui ${ }^{21}$, A. Sansom ${ }^{31}$, D. Scott ${ }^{33}$, S. Serjeant ${ }^{3}$, R. Sharp ${ }^{23}$, B. Sibthorpe ${ }^{25}$, D. J. B. Smith ${ }^{7}$, M. A. Thompson ${ }^{10}$, \\ R. Tuffs ${ }^{34}$, I. Valtchanov ${ }^{35}$, E. Van Kampen ${ }^{27}$, P. Van der Werf ${ }^{36}$, A. Verma ${ }^{37}$, J. Vieira ${ }^{8}$, and C. Vlahakis ${ }^{36}$
}

(Affiliations are available in the online edition)

Received 30 March 2010 / Accepted 26 April 2010

\begin{abstract}
We present colour-colour diagrams of detected sources in the Herschel-ATLAS science demonstration field from 100 to $500 \mu \mathrm{m}$ using both PACS and SPIRE. We fit isothermal modified black bodies to the spectral energy distribution (SED) to extract the dust temperature of sources with counterparts in Galaxy And Mass Assembly (GAMA) or SDSS surveys with either a spectroscopic or a photometric redshift. For a subsample of 330 sources detected in at least three FIR bands with a significance greater than $3 \sigma$, we find an average dust temperature of (28 \pm 8 ) K. For sources with no known redshift, we populate the colour-colour diagram with a large number of SEDs generated with a broad range of dust temperatures and emissivity parameters, and compare to colours of observed sources to establish the redshift distribution of this sample. For another subsample of 1686 sources with fluxes above $35 \mathrm{mJy}$ at $350 \mu \mathrm{m}$ and detected at 250 and $500 \mu \mathrm{m}$ with a significance greater than $3 \sigma$, we find an average redshift of $2.2 \pm 0.6$.
\end{abstract}

Key words. submillimeter: galaxies - Galaxy: evolution - galaxies: high-redshift

\section{Introduction}

The total intensity of the extragalactic background light at farinfrared (FIR) wavelengths has been measured with absolute photometry (Puget et al. 1996; Fixsen et al. 1998; Dwek et al. 1998). Deep surveys are now starting to resolve the cosmic FIR background into discrete sources with the fraction resolved varying with wavelength (see reviews in Blain et al. 2002; Hauser \& Dwek 2001; Lagache et al. 2005; Dye et al. 2009). Despite some successes, there are still large uncertainties about the nature and evolution of the submillimetre (submm) galaxy population, including their redshift distribution.

The Herschel Astrophysical Terahertz Large Area survey (H-ATLAS) is an open-time key program of the Herschel Observatory (Pilbratt et al. 2010) that will survey roughly $550 \mathrm{deg}^{2}$ of sky over $600 \mathrm{~h}$ of observations (Eales et al. 2010). To cover the widest possible area, H-ATLAS uses the maximum possible scan rate for the telescope at $60 \operatorname{arcsec~s}^{-1}$ in the parallel mode of PACS (Poglitsch et al. 2010) and SPIRE (Griffin et al. 2010). The observations cover five photometric bands with 100

* Herschel is an ESA space observatory with science instruments provided by European-led Principal Investigator consortia and with important participation from NASA. and $160 \mu \mathrm{m}$ data from PACS, and 250, 350, and $500 \mu \mathrm{m}$ data from SPIRE. The H-ATLAS fields include 1 field close to the northern Galactic pole (150 deg 2 ), 3 fields (each $36 \mathrm{deg}^{2}$ ) coinciding with the GAMA redshift survey fields (Driver et al. 2009), and 2 fields (total area of $250 \mathrm{deg}^{2}$ ) near the southern Galactic pole.

During the science demonstration phase (SDP), H-ATLAS observed $14.4 \mathrm{deg}^{2}$ in the GAMA-9 $\mathrm{h}$ field near the ecliptic plane to a $5 \sigma$ depths of 35-90 mJy. In this Letter we present the colour-colour diagrams derived from the five bands from 100 to $500 \mu \mathrm{m}$ for the H-ATLAS sources that are cross-identified in at least 3 of the SPIRE and PACS bands. We then discuss the dust temperature and redshift distributions based on certain assumptions about the spectral energy distribution (SED) and subject to selection effects associated with the sample selection.

\section{Colour-colour diagrams}

We use source catalogues generated for the H-ATLAS consortium by Rigby et al. (in prep.), derived from SPIRE and PACS maps presented in Pascale et al. (in prep.) and Ibar et al. (in prep.), respectively. The source catalogues are supplemented with cross-identification information from the GAMA survey (Driver et al. 2009) and SDSS DR-7 (Abazajian et al. 2009) as 
described in Smith et al. (in prep.). For the sources for which spectroscopic redshifts from GAMA or SDSS are not available, we use photometric redshifts generated using the ANNz neural network code (Collister \& Lahav 2004), trained with photometry from SDSS and UKIDSS LAS (Lawrence et al. 2007), and spectra from the GAMA spectroscopic survey (Driver et al. 2009), DEEP2 (Davis et al. 2007), zCOSMOS (Lilly et al. 2007), and the 2SLAQ-LRG (Cannon et al. 2006) survey.

In Fig. 1, we show the colour-colour plots of the H-ATLAS sources. We divide these plots in terms of colours based on either SPIRE only or SPIRE and PACS data. Our flux selection results in selecting 1686, 402, and 158 sources, from top to bottom of Fig. 1. For reference, the total ATLAS catalogue for this field contains $\sim 6600$ sources (Rigby et al., in prep.).

The colour-colour plots are filled with $10^{6}$ black-body spectra at a single dust temperature, $T_{\mathrm{d}}$, modified by a frequencydependent emissivity function $\epsilon_{v} \propto v^{\beta}$, where the flux density $f_{v}$ is

$f_{v}=\epsilon_{v} B_{v} \propto v^{3+\beta} /\left[\exp \left(\frac{h v}{k T_{\mathrm{d}}}\right)-1\right]$.

In generating these models, we consider uniform ranges of dust temperature from 10 to $60 \mathrm{~K}$, emissivity parameter $\beta$ from 0 to 2 , and redshift from 0 to 5 . The choice of $0<\beta<2$ compared to $1<\beta<2$ makes a minor difference since we also broaden the SED tracks in the colour diagram by adding an extra Gaussian standard deviation of $10 \%$ to the fluxes used to compute the model colour. This scatter accounts for the broadening of data in the colour-colour plane caused by flux uncertainties. As shown in Fig. 1, we find that the colour diagram of sources with fluxes only from SPIRE are well within the limits defined by the models we have considered. When we examine PACS colours, we find that some points lie outside the same set of tracks as used for the SPIRE-only colour diagram. While some of these outlier points may be cause by either the fractionally larger flux errors of PACS or contamination from a neighboring source, it is possible that some of these sources are not accurately described by our simple isothermal SED model, requiring for instance a second dust component (Dunne \& Eales 2001) or a more complex SED model.

When fitting a simple modified black-body model to the data, we must keep in mind that there is a partial degeneracy between $\beta$ and $T_{\mathrm{d}}$ and, more importantly, a perfect degeneracy between $T_{\mathrm{d}}$ and $z$. The peak of the SED is determined by the $v / T_{\mathrm{d}}$ term in the exponential, so that a measurement of the colours alone constrains only the ratio $(1+z) / T_{\mathrm{d}}$. However, assuming reasonable priors on the free parameters of the SED model (in our case, $\beta$ and $T_{\mathrm{d}}$ ) it is still possible to estimate a qualitative redshift distribution for our sample of sources. Alternatively, if secure redshifts are known from optical cross-identifications, we can determine the dust temperatures.

\section{Dust temperature distribution}

We consider the H-ATLAS source sample with detections at $3 \sigma$ in at least two bands and at $5 \sigma$ in one band of either PACS and SPIRE that have been robustly (reliability parameter $R_{\mathrm{LR}}>0.9$, Smith et al., in prep.) identified with GAMA or SDSS DR-7 galaxies. We also require that there is a known spectroscopic redshift from either GAMA or SDSS, or from the photometric redshift catalog (with $(1+z) / \sigma_{z}>5$ and $\left.z / \sigma_{z}>1\right)$ that was generated for this field and cross-identified with ATLAS sources (Smith et al., in prep.). We select 330 sources, which correspond
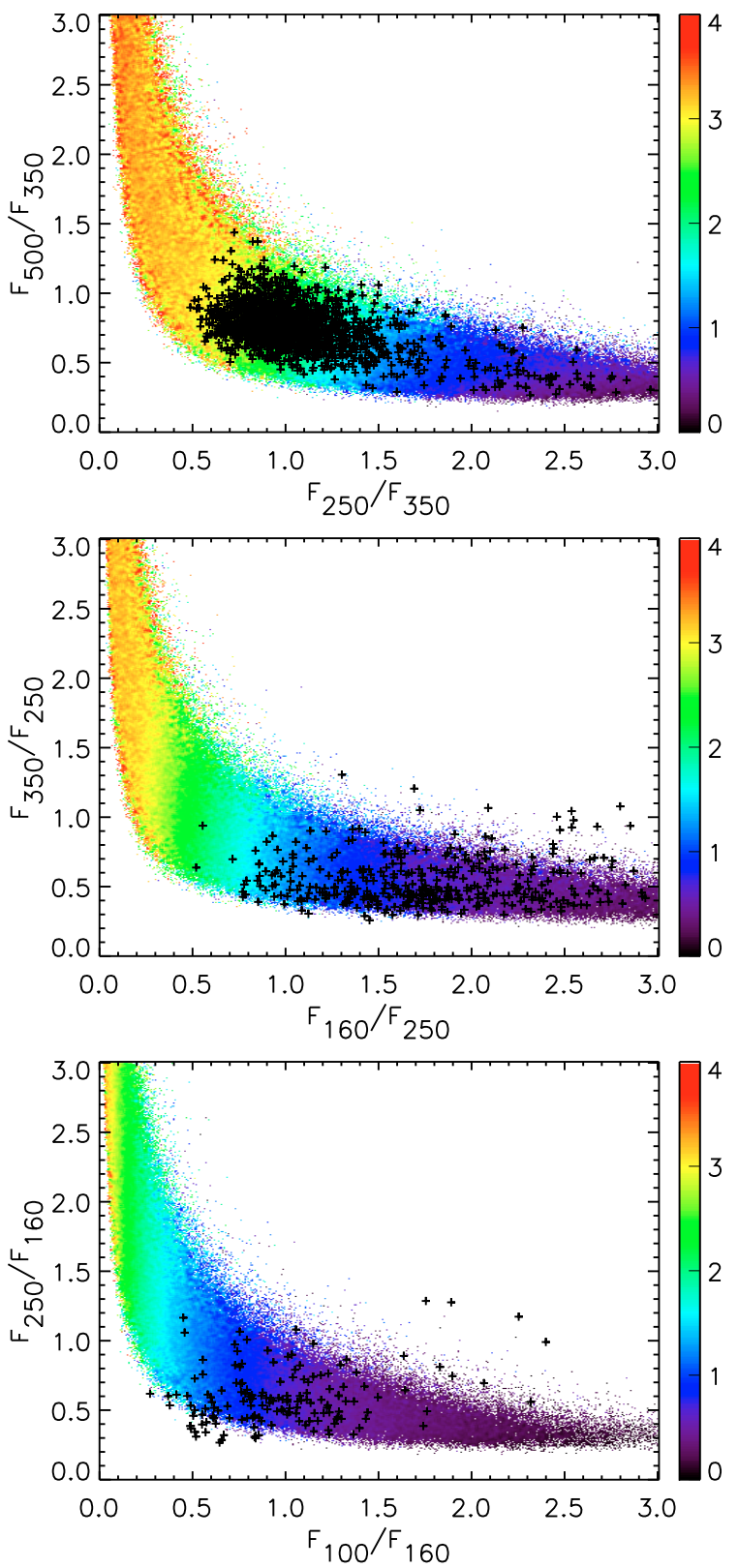

Fig. 1. Colour-colour diagram of SPIRE and PACS sources. The coloured background indicates the average redshift in these colourcolour spaces of our $10^{6}$ randomly generated model SEDs. In a) points plotted represent a sample of 1686 galaxies detected in all three SPIRE bands with a significance greater than $5 \sigma$ at $350 \mu \mathrm{m}(S>35 \mathrm{mJy})$ and greater than $3 \sigma$ at 250 and $500 \mu \mathrm{m}$ (corresponding to flux limits of roughly 21 and $27 \mathrm{mJy}$, respectively). In b) we show a sample of 402 galaxies detected at 160, 250, and $350 \mu \mathrm{m}$ and in c) we show a sample of 158 galaxies detected at 100,160 , and $250 \mu \mathrm{m}$. As in a), in both $\mathbf{b}$ ) and $\mathbf{c}$ ) we also select sources by imposing a $5 \sigma$ cut in the central wavelength of the colour plot and greater than $3 \sigma$ at other wavelengths.

to the low redshift subsample of the galaxies selected in the colour-colour diagrams. For each galaxy, we perform a single temperature fit from the above equation assuming $\beta=1.5$. We found that an isothermal SED model generally is a good fit to the 330 galaxies. Fitting a two-component SED model does not, on average, seem to provide a closer fit. The relatively good fit provided by the isothermal SED model is probably due to most of our sample consisting of low redshift galaxies and therefore that we do not probe much of the Wien part of the galaxy SED. 


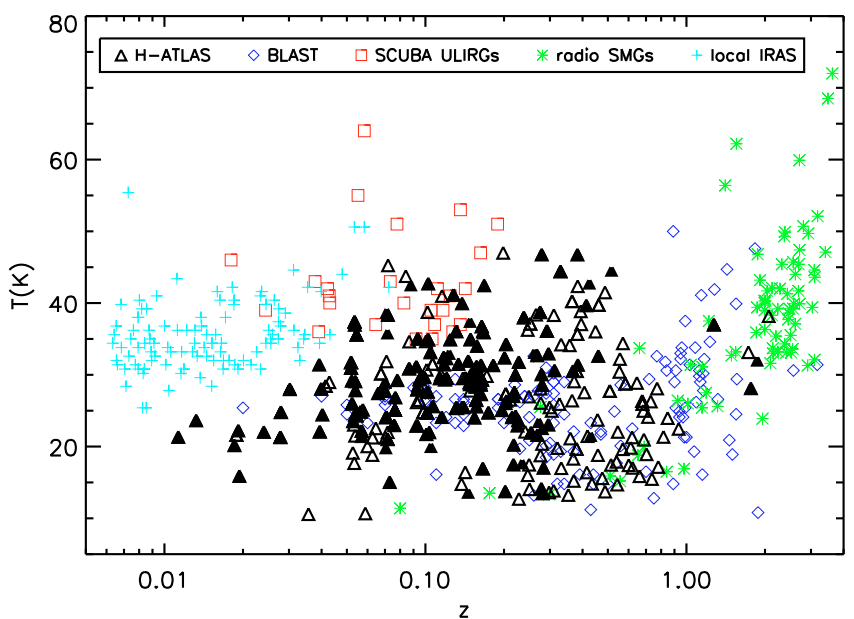

Fig. 2. Dust temperature as a function of redshift for $330 \mathrm{H}$-ATLAS sources reliably identified with GAMA and SDSS DR-7 galaxies having a known spectroscopic (black filled triangle) or a photometric (black empty triangle) redshift. The selected sources are $>5 \sigma$ detections in at least one band of either PACS or SPIRE with $3 \sigma$ flux measurements in at least 2 more bands. The isothermal spectral fits assume $\beta=1.5$. For other data plotted, see Sect. 3.

Table 1. Average dust temperatures as a function of redshift for the $330 \mathrm{H}$-ATLAS galaxies (Col. 2) and for all the data (Col. 4) presented in Figs. 2 and 3 (including H-ATLAS).

\begin{tabular}{ccccc}
\hline \hline \multirow{2}{*}{-range } & H-ATLAS & \multirow{2}{*}{$T_{\mathrm{d}}$} & $\begin{array}{c}\text { All data } \\
N_{\text {srcs }}\end{array}$ & All data $T_{\mathrm{d}}$ \\
\hline All $z$ & 330 & $28 \pm 8$ & 657 & $30 \pm 9$ \\
$0<z<0.1$ & 106 & $27 \pm 8$ & 235 & $32 \pm 9$ \\
$0.1<z<0.5$ & 186 & $29 \pm 8$ & 260 & $28 \pm 8$ \\
$0.5<z<1$ & 33 & $23 \pm 5$ & 67 & $24 \pm 7$ \\
$z>1$ & 5 & $35 \pm 4$ & 95 & $37 \pm 10$ \\
\hline
\end{tabular}

In Fig. 2, we summarize our results and compare the H-ATLAS dust temperatures with dust temperatures in the literature for a variety of sub-mm bright galaxies. These samples are: (i) the sources in BLAST detected above $5 \sigma$ in at least one of the BLAST bands with either a COMBO-17 (Wolf et al. 2004) or a SWIRE photometric redshift (Rowan-Robinson et al. 2008) and Spitzer-MIPS 70 and $160 \mu \mathrm{m}$ fluxes (Dye et al. 2009; $\beta=1.5$ fixed); (ii) local ULIRGS observed with SCUBA at 450 and $850 \mu \mathrm{m}$ and complemented with IRAS 60 and $100 \mu \mathrm{m}$ fluxes (Clements et al. 2010; $\beta$ varied); (iii) SCUBA sub-mm galaxies detected at better than $3 \sigma$ at $850 \mu \mathrm{m}$ and having redshifts determined from Keck-I spectroscopy (Chapman et al. 2005; $\beta=1.5$ fixed); and (iv) local IRAS-selected galaxies with 60 and $100 \mu \mathrm{m}$ fluxes complemented with SCUBA $850 \mu \mathrm{m}$ (Dunne et al. 2000; $\beta$ varied).

In Table 1, we list average dust temperatures as a function of redshift for several bins in redshift for both H-ATLAS only and all of the combined sub-mm galaxy samples, including H-ATLAS, plotted in Fig. 2. We do not find any evolution in the H-ATLAS dust temperature with redshift, though some evolution is inferred by BLAST measurements (Dye et al. 2009; Pascale et al. 2009), where sources at higher redshift had higher temperature in agreement with the radio-identified submillimeter-selected galaxies (SMGs) found with SCUBA (Chapman et al. 2005; Ivison et al. 2010; Kovacs et al. 2006; Coppin et al. 2008). While we find remarkable consistency between the average dust temperatures for our H-ATLAS sample

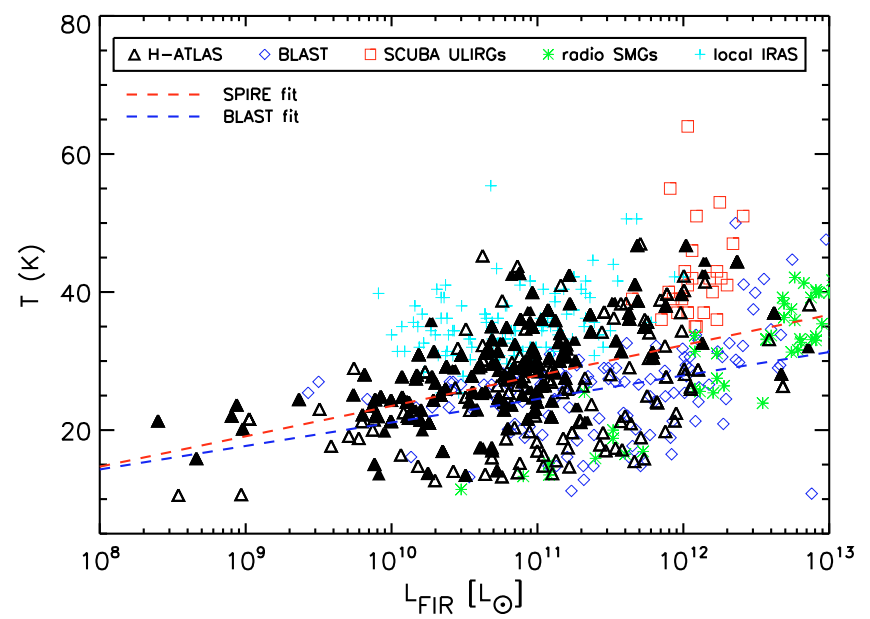

Fig. 3. Dust temperature as a function of the FIR luminosity for $330 \mathrm{H}$-ATLAS sources reliably identified with GAMA and SDSS DR-7 galaxies with a known spectroscopic (black filled triangle) or a photometric (black empty triangle) redshift. The FIR luminosity is obtained by fitting the PACS and SPIRE measurements with a modified black body (parameters are the dust temperature and the luminosity density, $\beta$ is fixed to 1.5) and this model SED is integrated between 8 and $1100 \mu \mathrm{m}$. We also indicate BLAST and H-ATLAS best-fit $T_{\mathrm{d}}-\log \left(L_{\mathrm{FIR}}\right)$ relation with a dashed blue line (from Dye et al. 2009) and a dashed red line, respectively.

as a function of redshift, these average values are not necessarily in agreement with other sub-mm galaxy subsamples in the literature, mostly due to selection effects. For example, the SCUBA ULIRGS have an average temperature of $(43 \pm 7) \mathrm{K}$. These sources were selected using IRAS $60 \mu \mathrm{m}$ data and IRAS selected sources are known to be biased towards higher temperatures. Optically-selected low-redshift sub-mm galaxies are known to have colder temperatures consistent with our findings (e.g., Willmer et al. 2009; Vlahakis et al. 2005). The large expected sample of sources from the $550 \mathrm{deg}^{2}$ of H-ATLAS will enable more detailed studies in the future, without the biases associated with selections of various sub-samples, including our own. While we show results here with $\beta=1.5$ fixed, when fitting for $\beta$ and $T_{\mathrm{d}}$ we found $\beta=1.4 \pm 0.1$, consistent with $\beta=1.3$ found in Dunne et al. (2000).

In Fig. 3, we plot the dust temperature versus FIR luminosity by integrating model SEDs between 8 and $1100 \mu \mathrm{m}$ for the H-ATLAS subsample. Luminosities for other samples are from the literature. Fitting for a relation of the form $T_{\mathrm{d}}=$ $T_{0}+\alpha \log \left(L_{\mathrm{FIR}} / L_{\odot}\right)$, we find $T_{0}=-20.5 \mathrm{~K}$ and $\alpha=4.4$ (see, Fig. 4). The value of $\log \left(L_{\mathrm{FIR}} / L_{\odot}\right)$ is on average $10.9 \pm 0.8$ for our sample. This relation is consistent with the BLAST data (Dye et al. 2009).

\section{Redshift distribution}

Since most of our sources lack redshifts, we consider another example application of our colour diagram and infer the statistical redshift distribution $N(z)$ for the source samples plotted in Fig. 1. We do this by first gridding the colour-colour plane along the SED tracks into redshift bins. We then convert the number of sources within a grid region of colours to a binned redshift distribution (Hughes et al. 2002). This method is equivalent to extracting the redshift probability distribution function for the whole sample if we had simply fitted SEDs to individual fluxes and taken the sum of the redshift probabilities of each source. While the redshift for an individual galaxy is largely uncertain, 


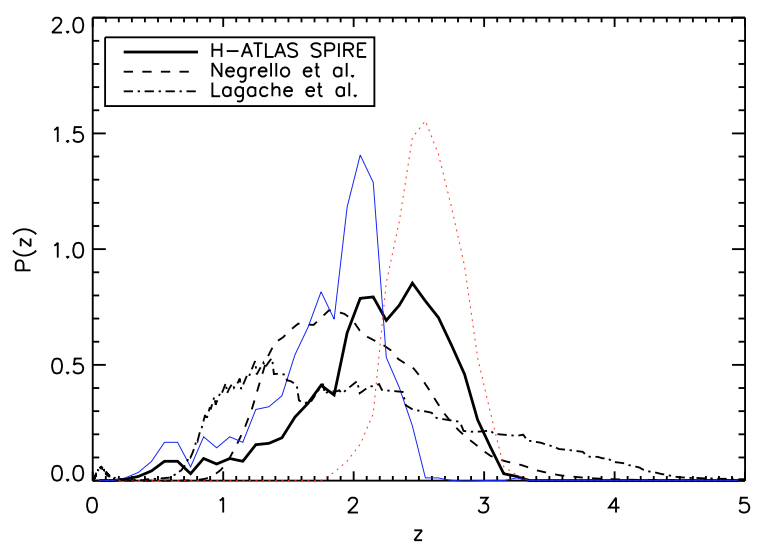

Fig. 4. Normalized redshift distribution (black thick solid line) of SPIRE sources in the colour-colour diagram of Fig. 1a, selected to satisfy the following criteria: $S_{350}>35 \mathrm{mJy}$ and at least $3 \sigma$ detections at 250 and $500 \mu \mathrm{m}$. The dashed line and dotted-dash line represent Negrello et al. (2007) and Lagache et al. (2004) models for the same flux selection. Both model curves have similar but not identical distributions, although Lagache model predicts a larger number of highredshift galaxies. In addition, we show $N(z)$ for the same subsample using a colour-cut of $S_{500} / S_{250}<0.75$ (blue thin solid line), and $>0.75$ (red thin dotted line). The distribution shown here is for 1686 sources, a subset of about $6600 \mathrm{H}$-ATLAS sources in the SDP field.

and sensitive to the SEDs used, the statistical redshift distribution we extract should be a reasonable estimate of the true distribution of the source sample.

Figure 4 shows the redshift distribution for SPIRE sources detected in all 3 bands (Fig. 1a). For the sample of 1686 sources with flux densities above $35 \mathrm{mJy}$ at $350 \mu \mathrm{m}$ and above $3 \sigma$ at 250 and $500 \mu \mathrm{m}$, we find the average redshift to be $2.2 \pm 0.6$. This is consistent with the average redshift of $2.4 \pm 0.4$ for the radioidentified sub-mm galaxies with SCUBA at $850 \mu \mathrm{m}$ followed up spectroscopically at Keck (Chapman et al. 2005), the interquartile redshift range of 1.8 to 3.1 for sources in SCUBA Half Degree Extragalactic Survey determined photometrically (Aretxaga et al. 2007), and a median redshift of greater than 1 for a subsample of $250 \mu \mathrm{m}$ sources as faint as $35 \mathrm{mJy}$ (Dunlop et al. 2009). Our distribution, however, has an average redshift that is higher than that of a larger sample of BLAST sources (Dye et al. 2009). This difference is not unexpected given the differences in the sample selection. We emphasize again that the distribution shown in Fig. 4 is for a subset of about $25 \%$ of the whole catalog with specific selection criteria that tend to favour the high redshift-end of the distribution. Based on crossidentifications with SDSS and GAMA, we find that $30 \%$ of the H-ATLAS sources are at $z<0.5$ in the SDP field (Smith et al., in prep.).

In addition to the total sample, we also make a colour-cut with $S_{500} / S_{250}>0.75$ and $<0.75$, in addition to the flux cut of $35 \mathrm{mJy}$ at $350 \mu \mathrm{m}$. By imposing this colour-cut, we distinguish a high redshift population with redder colours from a low redshift population. We find average redshifts of $2.6 \pm 0.3$ and $1.8 \pm 0.4$ for sources with $S_{500} / S_{250}>0.75$ and $<0.75$, respectively. While we do not show the redshift distributions separately for the samples in Figs. 1b and c, the average redshift in these cases is $z=0.8 \pm 0.3$ for sources detected in 160,250 , and $350 \mu \mathrm{m}$ with a flux cut of $35 \mathrm{mJy}$ at $250 \mu \mathrm{m}$, and $z=0.7 \pm 0.3$ for sources detected in 100,160 , and $250 \mu \mathrm{m}$ with a flux cut of $\simeq 100 \mathrm{mJy}$ at $160 \mu \mathrm{m}$. As is clear from Fig. 1, we find that lower redshift sources are more likely to be detected at shorter wavelengths, while bright sources seen in longer SPIRE bands are likely to be on average at high redshifts. Some of the bright $500 \mu \mathrm{m}$ sources that we detect, especially with a colour selection selecting red galaxies in the sub-mm, may also have their fluxes magnified by lensing and will be among the results forthcoming from $\mathrm{H}$ ATLAS (Negrello et al., in prep.).

\section{Conclusions}

We have discussed the spectral energy distribution of sub-mm galaxies in a $14 \mathrm{deg}^{2}$, subset of the full H-ATLAS survey. We have used the colours in 5 bands to measure the dust temperature for the sub-sample of sources with known redshifts. We have also derived a qualitative estimate of the statistical redshift distribution of bright $350 \mu \mathrm{m}$ selected galaxies assuming broad priors in temperature and the dust emissivity. We found that the average dust temperature of $\mathrm{H}$-ATLAS sources is $28 \pm 8 \mathrm{~K}$, while $350 \mu \mathrm{m}$ sources with fluxes above $35 \mathrm{mJy}$ have an average redshift of $2.2 \pm 0.6$.

Acknowledgements. Amblard, Barton, Cooray, Leeuw, Serra and Temi acknowledge support from NASA funds for US participants in Herschel through JPL. Funding for the SDSS and SDSS-II has been provided by the Alfred P. Sloan Foundation, the Participating Institutions, the National Science Foundation, the US Department of Energy, the National Aeronautics and Space Administration, the Japanese Monbukagakusho, the Max Planck Society, and the Higher Education Funding Council for England. The SDSS Web Site is http://www . sdss.org/. The SDSS is managed by the Astrophysical Research Consortium for the Participating Institutions.

The UKIDSS project is defined in Lawrence et al. (2007).

\section{References}

Abazajian, K., Adelman-McCarthy, J., Aguüeros, M., et al. 2009, ApJS, 182, 543

Aretxaga, I., Hughes, D. H., Coppin, K., et al. 2007, MNRAS, 379, 1571

Blain, A. W., Smail, I., Ivison, R. J., et al. 2002, Phys. Rept., 369, 111

Cannon, R., Drinkwater, M., Edge, A., et al. 2006, MNRAS, 372, 425

Chapman, S. C., Blain, A. W., Smail, I., et al. 2005, ApJ, 622, 772

Clements, D. L., Dunne, L., \& Eales, S. 2010, MNRAS, 403, 274

Collister, A. A., \& Lahav, O. 2004, PASP, 116, 345

Coppin, K., Halpern, M., Douglas, S., et al. 2008, MNRAS, 384, 1597

Davis, M., Guhathakurta, P., Konidaris, N. P., et al. 2007, ApJ, 660, L1

Driver, S. P., Norberg, P., Baldry, I. K., et al. 2009, Astron. Geophys., 50, 5.12

Dunlop, J. S., Ade, P. A. R., Bock, J. J., et al. 2009, MNRAS, submitted [arXiv: 0910.3642]

Dunne, L., \& Eales, S. 2001, MNRAS, 327, 697

Dunne, L., Eales, S., Edmunds, M., et al. 2000, MNRAS, 315, 115

Dwek, E., Arendt, R. G., Hauser, M. G., et al. 1998, ApJ, 508, 106

Dye, S., Ade, P. A. R., Bock, J. J., et al. 2009, ApJ, 703, 285

Eales, S., Dunne, L., Clements, D., et al. 2010, PASP, 122, 499

Fixsen, D. J., Dwek, E., Mather, J. C., et al. 1998, ApJ, 508, 123

Griffin, M. J., et al. 2010, A\&A, 518, L3

Hauser, M. G., \& Dwek 2001, ARA\&A, 39, 249

Hughes, D. H., Aretxaga, I., Chapin, E. L., et al. 2002, MNRAS, 335, 871

Ivison, R. J., Alexander, D. M., Biggs, A. D., et al. 2010, MNRAS, 402, 245

Kovacs, A., Chapman, S. C., Dowell, C. D., et al. 2006, ApJ, 650, 592

Lagache, G., Dole, H., Puget, J.-L., et al. 2004, ApJS, 154, 112

Lagache, G., Puget J.-L., \& Dole, H. 2005, ARA\&A, 43, 727

Lawrence, A., Warren, S. J., Almaini, O., et al. 2007, MNRAS, 379, 1599

Lilly, S. J., Le Fèvre, O., Renzini, A., et al. 2007, ApJS, 172, 70

Negrello, M., Perrotta, F., González-Nuevo, J., et al. 2007, MNRAS, 377, 1557

Pascale, E., Ade, P. A. R., Bock, J. J., et al. 2009, ApJ, 707, 1740

Pilbratt, G. L., et al. 2010, A\&A, 518, L1

Poglitsch, A., et al. 2010, A\&A, 518, L2

Puget, J.-L., Abergel, A., Bernard, J.-P., et al. 1996, A\&A, 308, L5

Rowan-Robinson, M., Babbedge, T., Oliver, S., et al. 2008, MNRAS, 386, 697

Vlahakis, C., Dunne, L., \& Eales, S. 2005, MNRAS, 364, 1253

Willmer, C. N. A., Rieke, G. H., Le Floc'h, E., et al. 2009, AJ, 138, 146

Wolf, C., Meisenheimer, K., Kleinheinrich, M., et al. 2004, A\&A, 421, 913

Page 5 is available in the electronic edition of the journal at http: //www . aanda . org 
1 Dept. of Physics \& Astronomy, University of California, Irvine, CA 92697, USA, e-mail: amblard@uci .edu

2 Astrophysics Branch, NASA Ames Research Center, Mail Stop 2456, Moffett Field, CA 94035, USA

3 Dept. of Physics and Astronomy, The Open University, Milton Keynes, MK7 6AA, UK

${ }^{4}$ School of Physics and Astronomy, Cardiff University, The Parade, Cardiff, CF24 3AA, UK

5 Sterrenkundig Observatorium, Universiteit Gent, Krijgslaan 281 S9, 9000 Gent, Belgium

6 Astrophysics Research Institute, Liverpool John Moores University, 12 Quays House, Egerton Wharf, Birkenhead, CH41 1LD, UK

7 School of Physics and Astronomy, University of Nottingham, University Park, Nottingham NG7 2RD, UK

8 Caltech, 249-17, Pasadena, CA 91125, USA

9 Jet Propulsion Laboratory, Pasadena, CA 91109; Department of Astronomy, California Institute of Technology, Pasadena, CA 91125, USA

10 Centre for Astrophysics Research, Science and Technology Research Centre, University of Hertfordshire, Herts AL10 9AB, UK

11 Laboratoire d'Astrophysique de Marseille, UMR6110 CNRS, 38 rue F. Joliot-Curie, 13388 Marseille, France

12 INAF - Osservatorio Astronomico di Padova, Vicolo Osservatorio 5, 35122 Padova, Italy

13 ETH Zurich, Insitute for Astronomy, HIT J12.3, 8093 Zurich, Switzerland

14 Instituto de Astrofísica de Canarias, C/Vía Láctea s/n, 38200 La Laguna; Departamento de Astrofísica, Universidad de La Laguna (ULL), 38205 La Laguna, Tenerife, Spain

15 Astrophysics Group, Imperial College, Blackett Laboratory, Prince Consort Road, London SW7 2AZ, UK

16 Sydney Institute for Astronomy, School of Physics, University of Sydney, NSW 2006, Australia

17 SUPA, School of Physicsand Astronomy, University of St. Andrews, North Haugh, St. Andrews, KY169SS, UK

18 Scottish Universities Physics Alliance, Institute for Astronomy, University of Edinburgh, Edinburgh, EH9 3HJ, UK
19 Infrared Processing and Analysis Center, California Institute of Technology, 770 South Wilson Av, Pasadena, CA 91125, USA

20 NASA Goddard Space Flight Center, Greenbelt, MD 20771, USA

21 Scuola Internazionale Superiore di Studi Avanzati, via Beirut 2-4, 34151 Triest, Italy

22 Instituto de Física de Cantabria (CSIC-UC), Santander, 39005, Spain

23 Anglo-Australian Observatory, PO Box 296, Epping, NSW 1710, Australia

24 Instituto Nacional de Astrofísica, Óptica y Electrónica (INAOE), Luis Enrique Erro No. 1, Tonantzintla, Puebla, C.P. 72840, Mexico

25 UK Astronomy Technology Center, Royal Observatory Edinburgh, Edinburgh, EH9 3HJ, UK

26 Institut d'Astrophysique Spatiale (IAS), Bâtiment 121, 91405 Orsay; and Université Paris-Sud 11 and CNRS (UMR 8617), France

27 European Southern Observatory, Karl-Schwarzschild-Strasse 2 85748, Garching bei Munchen, Germany

28 Astronomy Centre, University of Sussex, Falmer, Brighton, BN1 9QH, UK

29 Space Science \& Technology Department CCLRC RAL, Rutherford Appleton Laboratory Oxon, OX11 0QX, UK

30 Institute for Space Imaging Science, University of Lethbridge, Lethbridge, Alberta, T1K 3M4, Canada

31 Jeremiah Horrocks Institute, University of Central Lancashire, Preston, Lancs PR1 2HE, UK

32 University of Padova, Department of Astronomy, Vicolo Osservatorio 3, 35122 Padova, Italy

33 Dept. of Physics \& Astronomy, University of British Columbia, 6224 Agricultural Road, Vancouver, B.C. V6T 1Z1, Canada

34 Max Planck Institute for Nuclear Astrophysics (MPIK), Saupfercheckweg 1, 69117 Heidelberg, Germany

35 Herschel Science Centre, ESAC, ESA, PO Box 78, Villanueva de la Cañada, 28691 Madrid, Spain

36 Sterrewacht Leiden, Leiden University, PO Box 9513, 2300 RA Leiden, The Netherlands

37 Oxford Astrophysics, Denys Wilkinson Building, University of Oxford, Keble Road, Oxford, OX1 3RH, UK 